University of Nebraska - Lincoln

DigitalCommons@University of Nebraska - Lincoln

January 1980

\title{
A Graphical Method for Calculating First Order Transition Matrices for Open-Shell Atoms in the Random Phase Approximation
}

Anthony F. Starace

University of Nebraska-Lincoln, astarace1@unl.edu

Siamak Shahabi

University of Nebraska - Lincoln

Follow this and additional works at: https://digitalcommons.unl.edu/physicsstarace

Part of the Physics Commons

Starace, Anthony F. and Shahabi, Siamak, "A Graphical Method for Calculating First Order Transition Matrices for Open-Shell Atoms in the Random Phase Approximation" (1980). Anthony F. Starace Publications. 140.

https://digitalcommons.unl.edu/physicsstarace/140

This Article is brought to you for free and open access by the Research Papers in Physics and Astronomy at DigitalCommons@University of Nebraska - Lincoln. It has been accepted for inclusion in Anthony F. Starace Publications by an authorized administrator of DigitalCommons@University of Nebraska - Lincoln. 


\title{
A Graphical Method for Calculating First Order Transition Matrices for Open-Shell Atoms in the Random Phase Approximation
}

\author{
Anthony F. Starace and Siamak Shahabi \\ Behlen Laboratory of Physics, University of Nebraska-Lincoln, Lincoln, Nebraska 68588, USA
}

\begin{abstract}
A graphical procedure is presented for calculating first order transition matrices for a general (open-shell) atom. The first order transition matrix may be used to calculate matrix elements of a general one-body operator of rank $\lambda$ in orbital space and $\sigma$ in spin space. In the random phase approximation we obtain a set of $N$ $+N^{\prime}$ coupled differential equations for $N$ final state radial functions and $N^{\prime}$ initial state radial functions which completely determine the first order transition matrix for an atomic system having $N$ final state channels. (The relation of $N^{\prime}$ to $N$ is dependent on the atomic system studied.) These $N+N^{\prime}$ differential equations reduce to familiar forms in the following cases: (1) When initial state correlations are ignored, we obtain the $N$ coupled differential equations of the Close-Coupling Approximation; (2) When the atom has only closed subshells we obtain $N^{\prime}=N$ and the $2 N$ coupled differential equations are those obtained in the Chang-Fano version of the Random Phase Approximation.
\end{abstract}

\section{Introduction}

Current theoretical understanding of closed-shell atom photoionization cross sections has been based on the Random Phase Approximation (RPA) [1, 2], which implies that, in addition to the usual final state interactions, virtual excitations of pairs of valence electrons have an important influence on these cross sections. The importance of the electron correlations included in the random phase approximation have been confirmed for closed shell atoms by other theoretical methods, especially the Many-Body Perturbation Theory [3] and the RMatrix Theory $[4,5]$. The theoretical understanding of the influence of the electron correlations on open-shell atom photoionization cross sections is less developed. This is due to the greater theoretical difficulty of dealing with atoms that are not spherically symmetric and which thus have a great number of final state channels. While RPA theories have been developed to treat open-shell atoms [6-9] these have been given in the form of matrix or integral equations which require the use of large numbers of basis functions for their solution.

In what follows we outline a new graphical method for calculating the first order transition matrix $[10,11]$ for a general (open-shell) atom. This matrix may be used to calculate matrix elements of a general one-body operator having ranks $\lambda$ and $\sigma$ in orbital and spin space respectively. In particular we may calculate the electric dipole matrix elements (having $\lambda=1$ and $\sigma=0$ ) which are needed to calculate photoionization cross-sections. The graphical method greatly simplifies the treatment of antisymmetrization and of angular momentum algebra and in addition affords a physical insight into the physical processes involved that is similar to that afforded by the diagrams of ordinary many-body perturbation theory. In the RPA we show that the first order transition matrix for an atom having $N$ fi- nal state channels is determined by a set of $N$ final state radial functions and $N^{\prime}$ initial state radial functions which satisfy $N+$ $N^{\prime}$ coupled differential equations. (The relation of $N^{\prime}$ to $N$ depends on the particular atom studied.) Large numbers of basis functions are thus not needed. The $\left(N+N^{\prime}\right)$ coupled differential equations reduce to familiar forms in the following limiting cases: (1) When initial state correlations are ignored we obtain the $N$ coupled differential equations of the Close-Coupling Approximation [12] ; (2) When the atom has only closed shells, $N=N^{\prime}$ and we obtain the $2 N$ coupled differential equations of the Chang-Fano version of the RPA [11, 13]. A detailed description of our theoretical methods will be published elsewhere [14].

\section{The graphical method}

\subsection{Definition of the transition matrix}

Suppose that in a certain transition an $N$-electron atom may be described by an initial state $\langle i|$ and a final state $|f\rangle$. We define the first order transition matrix $\left\langle\mathbf{r}_{N}^{\prime}|\Gamma| \mathbf{r}_{N}\right\rangle$ as $[10,11]$

$$
\begin{aligned}
& \left\langle\mathbf{r}_{N}^{\prime}|\boldsymbol{\Gamma}| \mathbf{r}_{N}\right\rangle \\
& \quad \equiv N \prod_{j=1}^{N-1} \iint \mathrm{d}^{3} \mathbf{r}_{j}^{\prime} \mathrm{d}^{3} \mathbf{r}_{j}\left\langle\mathbf{r}_{1}^{\prime} \ldots \mathbf{r}_{N}^{\prime} \mid f\right\rangle\left\langle i \mid \mathbf{r}_{1} \ldots \mathbf{r}_{N}\right\rangle \delta\left(\mathbf{r}_{j}^{\prime}-\mathbf{r}_{j}\right)(1)
\end{aligned}
$$

Here each $\mathbf{r}$ includes also the spin coordinate of the corresponding electron. Once the first order transition matrix is determined, the matrix element of any one-body operator $\mathbf{O}^{\lambda, \sigma}(\mathbf{r})$ of rank $\lambda$ in orbital space and rank $\sigma$ in spin space is determined by the following one-electron integral:

$$
\left\langle i\left|\mathbf{O}^{\lambda, \sigma}\right| f\right\rangle=\iint \mathrm{d}^{3} \mathbf{r}_{N}^{\prime} \mathrm{d}^{3} \mathbf{r}_{N}\left\langle\mathbf{r}_{N}^{\prime}|\boldsymbol{\Gamma}| \mathbf{r}_{N}\right\rangle \mathbf{O}^{\lambda, \sigma}\left(\mathbf{r}_{N}\right) \delta\left(\mathbf{r}_{N}^{\prime}-\mathbf{r}_{N}\right)
$$

The outer product $|f\rangle\langle i|$ must satisfy the following dynamical equation if the atom is described by the Hamiltonian $H$ :

$$
H|f\rangle\langle i|-| f\rangle\langle i|H=\hbar \omega| f\rangle\langle i|
$$

Here $\hbar \omega$ is the transition energy, $E_{f}-E_{i}$. If equation (3) is integrated over $N-1$ coordinates then on the right hand side we obtain $\hbar \omega\left\langle\mathbf{r}_{N}{ }_{N}|\Gamma| \mathbf{r}_{N}\right\rangle$, while on the left we obtain partially integrated matrix elements of $H$ between the initial and final states. The result is a consistency equation which the orbitals defining $|f\rangle$ and $\langle i|$ must satisfy.

We suppose that the object of a calculation is to obtain the matrix elements of an operator $\mathbf{O}^{\lambda, \sigma}$ according to equation (2). To do this we must first specify the form of $|i\rangle$ and $\langle f|$ in terms of certain unknown radial wavefunctions, and then use the dynamical equation (3) to determine these unknown radial functions. We shall assume in this paper that the atom has an outer 
configuration $p^{q}$, where $2 \leq q \leq 6$, although this is not a restriction on the theory. Since we are concerned with one-body transition operators we assume the final state to have the form

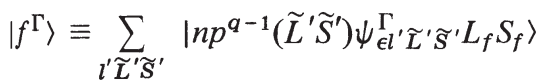

Here $\Gamma$ specifies a particular final state set of quantum numbers, $\psi^{\Gamma} \epsilon_{l^{\prime} \tilde{L}^{\prime} \tilde{S}^{\prime}}(r)$ is an unknown radial wavefunction for the excited electron, and the ionic core wavefunctions are assumed to be Hartree-Fock wavefunctions. The initial state is assumed to have the following correlated form:

$\langle i| \equiv\left\langle n p^{q} L_{i} S_{i}\right|+\sum_{\substack{l_{\phi} \bar{L} \bar{S} \\ L_{p} S_{p}}} b_{\bar{L} \bar{S}, L_{P} S_{P}}\left\langle n p^{q-2}(\bar{L} \bar{S}) \phi_{a} \phi_{b}\left(L_{P} S_{P}\right) L_{i} S_{i}\right|$

Here $\phi_{a}$ and $\phi_{b}$ are the radial wavefunctions for a pair of electrons excited out of the initial state configuration, each assumed to have an orbital angular momentum $l_{\phi}$. The orbitals of the configurations $p^{q}$ and $p^{q-2}$ are taken to be Hartree-Fock orbitals. Substituting equations (4) and (5) in equation (3) and integrating over $N-1$ coordinates we obtain equations that enable us to determine the unknown functions $\psi^{\Gamma} \epsilon_{l^{\prime} \tilde{L}^{\prime} \tilde{S}^{\prime}}(r)$ and a particular linear combination of $\phi_{a}(r)$ and $\phi_{b}(r)$ that we need to obtain the matrix elements in equation (2).

\subsection{The graphical rules}

The graphical method that we introduce has been developed to calculate the matrix elements arising when equation (3) is integrated over $N-1$ coordinates. The right hand side of equation (3) may be evaluated simply from the graph in Figure 1. On the left hand side of equation (3), the scalar one-body operators in $H$ lead to diagrams similar to those of Figure 1 and thus are not shown. The two-body Coulomb operator in $H$ leads to more complicated diagrams: the first term on the left of equation (3) leads to final state correlation diagrams, shown in Figure 2, while the second term on the left of equation (3) leads to initial state correlation diagrams, shown in Figure 3. Each of the direct interaction diagrams in Figures 2 and 3 has a corresponding exchange diagram which, for brevity's sake, is not shown.

The rules for writing down the graphs in Figures (1)-(3) are a slight modification of those in Section 6.1 of the review article of Briggs [15]. That is, we use his graphical notation for state vectors and Coulomb interaction operators. In contracting the state vectors with the Coulomb interaction operator, however, we have left a gap between a $p$-electron and an $l^{\prime}$ electron in each diagram to indicate that the $N$ th coordinate is not integrated over. The vertical bars on these electron lines indicate the radial wavefunctions (from the left-hand and righthand state vectors) which are not integrated over. The square boxes on some nodes indicate appropriate coefficients of fractional parentage, and the cross on the interaction line $k$ in Figure 2(a), for example, indicates a partially integrated reduced matrix element [11]

$$
\begin{aligned}
& (-1)^{k}\left(l^{\prime}\left\|V^{k}\left(\chi_{n p}, \chi_{n p} ; r\right)\right\| l^{\prime \prime}\right) \\
& \quad \equiv(-1)^{k}\left(l^{\prime}\left\|C^{(k)}\right\| l^{\prime \prime}\right)\left(1\left\|C^{(k)}\right\| 1\right) \int_{0}^{\infty} \mathrm{d} r^{\prime} \chi_{n p}\left(r^{\prime}\right) \chi_{n p}\left(r^{\prime}\right) \frac{r_{<}^{k}}{r_{>}^{k+1}}
\end{aligned}
$$

where

$$
\left(l^{\prime}\left\|C^{(k)}\right\| l^{\prime \prime}\right) \equiv(-1)^{l^{\prime}}\left[l^{\prime}\right]^{1 / 2}\left[l^{\prime \prime}\right]^{1 / 2}\left(\begin{array}{lll}
l^{\prime} & k & l^{\prime \prime} \\
0 & 0 & 0
\end{array}\right)
$$

and where $[l]=2 l+1$ and $r_{<}=\min \left(r, r^{\prime}\right)$.

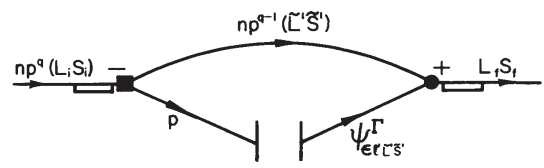

(a)

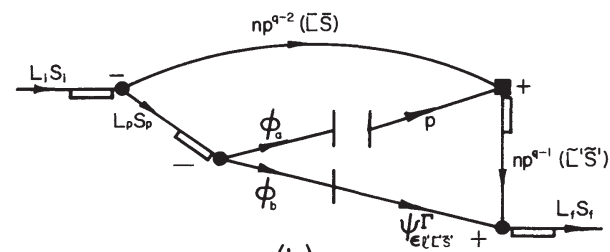

(b)

Figure 1. Graphical representation of the first order transition matrix for final and initial atomic state vectors represented by equations (4) and (5), respectively. Diagram (a) results from the initial state configuration $n p^{q}$, while diagram (b) results from the initial state configuration $n p^{q-2} \phi_{a} \phi_{b}$.
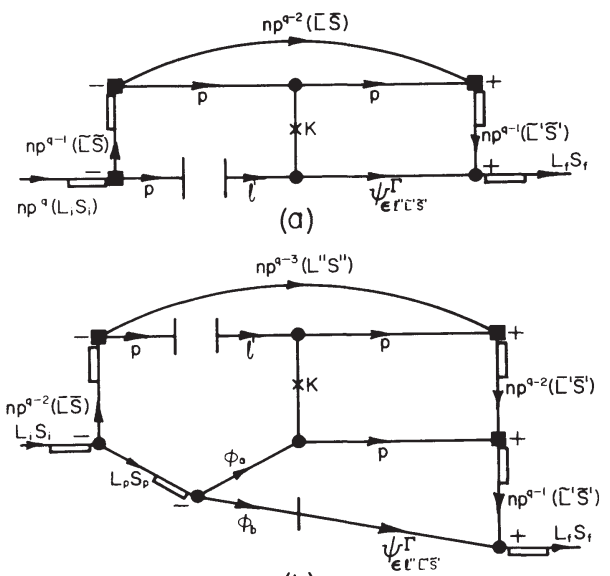

(b)

Figure 2. Final state direct interaction diagrams resulting from integrating the first term on the left hand side of equation (3) over $N-1$ coordinates.
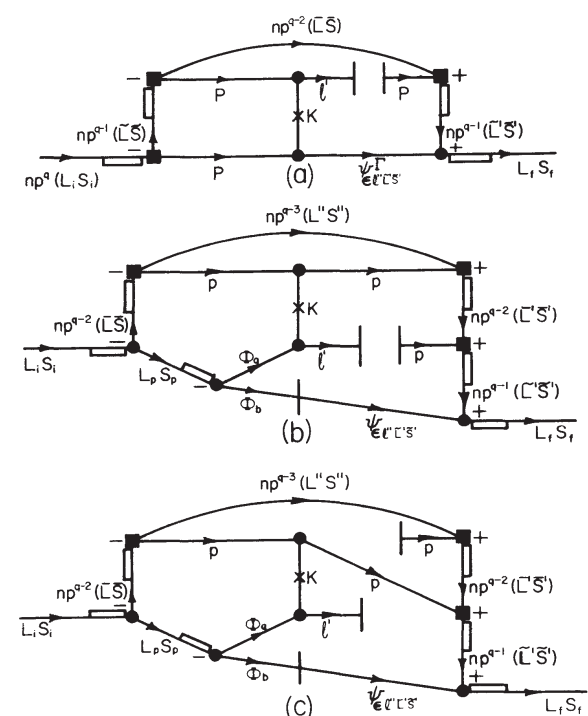

Figure 3. Initial state direct interaction diagrams resulting from integrating the second term on the left hand side of equation (3) over $N-1$ coordinates. 
In those diagrams involving the correlated ground state terms (i.e., those in Figures 1(b), 2(b), 3(b), and 3(c)) there are a number of additions to the rules in Section 6.1 of Briggs' review [15]. (1) There is an overlap integral $\left(\phi_{b} \mid \psi_{l^{\prime} \tilde{L}^{\prime} \tilde{S}^{\prime}}^{\Gamma}\right)$ indicated by the single vertical bar between the corresponding electron lines. This overlap implies a delta function $\delta\left(l_{\phi}, l^{\prime \prime}\right)$. (2) When the labels $a$ and $b$ are switched, we obtain diagrams identical in form to those shown but differing by the phase ($1)^{L p+S p}$. (3) Briggs' weight factor rule (i.e., rule (viii) in Section 6.1 of [15]) must be modified in order to include certain exclusion-principle-violating diagrams that are characteristic of the RPA. This modification is discussed later below.

Each of the diagrams in Figures 1-3 has four open lines: the two one-electron lines representing the $N$ th electron, whose coordinates are not integrated over, and the two lines indicating the initial and final orbital and spin angular momenta $L_{i} S_{i}$ and $L_{f} S_{f}$. These latter two open lines imply a dependence on magnetic quantum numbers. We can factor out this dependence and obtain a closed diagram in two steps: (1) We expand the two one electron states $p$ and $l^{\prime}$ in states of orbital angular momentum $\lambda$ and spin angular momentum $\sigma$; (2) we then factor out the dependence on magnetic quantum numbers by a graphical step analogous to the Wigner-Eckart Theorem. Thus each of the graphs in Figures 1-3 is transformed according to the rule shown in Figure 4 . The symbols $\psi_{a}\left(r_{a}\right)$ and $\psi_{b}\left(r_{b}\right)$ represent the radial wavefunctions associated with the horizontal bars in the dangling lines on the left side of the equation shown in Figure 4. The double tensor operator $W^{\lambda, \sigma}$ in Figure 4 is defined by [11] :

$$
\begin{aligned}
& \left(l_{b}, \hat{r}_{b}\left|W_{m_{\lambda}, m_{\sigma}}^{\lambda, \sigma}\right| l_{a}, \hat{r}_{a}\right)=\sum_{m_{1 / 2}^{a} m_{1 / 2}^{b}} \chi^{*}\left(\frac{1}{2}, m_{1 / 2}^{a}\right) \chi\left(\frac{1}{2}, m_{1 / 2}^{b}\right) \\
& \times(-1)^{1 / 2-m_{1 / 2}^{a}}\left(\frac{1}{2} m_{1 / 2}^{b}, \frac{1}{2}-m_{1 / 2}^{a} \mid \frac{1}{2} \frac{1}{2} \sigma m_{\sigma}\right) \\
& \times \sum_{m_{a^{m_{b}}}} Y_{l_{a} m_{a}}^{*}\left(\hat{r}_{a}\right) Y_{l_{b} m_{b}}\left(\hat{r}_{b}\right) \\
& \times(-1)^{l_{a}-m_{a}}\left(l_{b} m_{b}, l_{a}-m_{a} \mid l_{a} l_{b} \lambda m_{\lambda}\right)
\end{aligned}
$$

In equation (8) the $\chi^{\prime}$ s represent two component spin wavefunctions and the $Y^{\prime}$ 's represent spherical harmonics.

The closed diagrams resulting from application of the rule in Figure 4 to the diagrams in Figures 1-3 may now be transformed to the pure angular momentum diagrams of Jucys et al. [16] by application of rules (i)-(v) in Section 4.2 of Brigg's

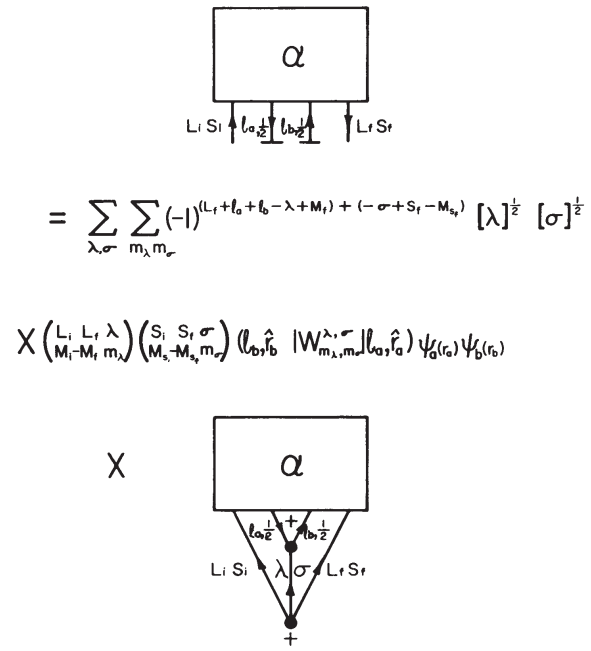

Figure 4. Graphical rule for removing the dependence on magnetic quantum numbers of diagrams such as those in Figures 1-3. review [15]. (Note that rule (iv) of Briggs [15] must be amended as follows: one changes the sign of all nodes except those on the $\lambda, \sigma$ interaction line in both the orbital and spin diagrams.) The resulting diagrams can be manipulated according to the rules of Jucys et al. [16] and reduced to algebraic expressions.

\subsection{The random phase approximation}

So far our treatment has been exact, given our choice of initial and final states in equations (4) and (5) and our selection of interactions, shown in Figures 2 and 3 (and including the exchange interactions corresponding to those shown). Some of the diagrams represent rather complicated interactions, particularly those involving the interactions of the correlated ground state function $\phi_{a}$ with the $n p^{q-2}$ core (i.e., diagrams 2(b), 3(b), and $3(\mathrm{c})$ ). These latter interactions are difficult to treat primarily because the presence of coefficients of fractional parentage does not permit the summations over $L^{\prime \prime}$ and $S^{\prime \prime}$ to be performed analytically. We therefore make an approximation to these diagrams which, in the closed shell case, results precisely in the correct RPA matrix elements. The approximation is this: we assume that when the correlated ground state function $\phi_{a}$ interacts with the $n p^{q-2}$ core, there is no exchange of orbital or spin angular momentum with the core. Furthermore, the weight factor for this interaction is not $(q-2)$, as given by Briggs' rules, but rather 6, corresponding to the fact that the exclusion principle is ignored and all core state magnetic quantum numbers are summed over.

The graphical expression of our RPA rule, which we apply to diagrams 2(b), 3(b), and 3(c), is shown in Figure 5. The diagram at the top of Figure 5 shows the general structure of the diagrams 2(b), 3(b), and 3(c). The block labeled $\beta$ includes in particular the interaction of the one electron orbital $p, 1 / 2$ with the correlated ground state function $\phi_{a}$. The middle part of Figure 5 shows an exact expansion (in orbital and spin angular momenta $\lambda^{\prime}$ and $\sigma^{\prime}$ ) of the one-electron orbital $p, 1 / 2$ before and after interaction with the block labeled $\beta$. The last line in Figure 5 shows our approximation to this exact expansion: we have kept only the terms with $\lambda^{\prime}=\sigma^{\prime}=0$, and we have multiplied by the weight factor $6=[1][1 / 2]$ rather than $q-2$. This approximation gives the delta functions $\delta_{\bar{L} \bar{L}^{-}}$, and $\delta_{\bar{S} \bar{S}}$, and permits an analytic
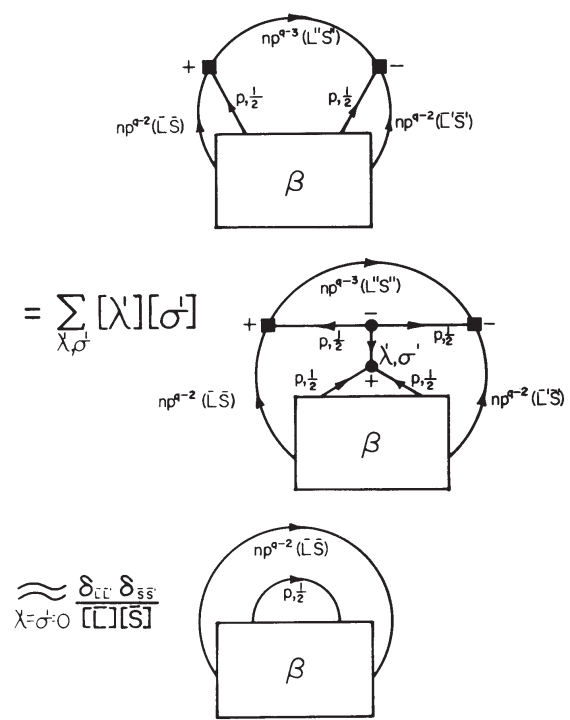

Figure 5. Graphical representation of our random phase approximation: we assume the $n p^{q-2}$ core exchanges zero orbital and spin angular momentum (i.e., $\lambda^{\prime}=\sigma^{\prime}=0$ ) with the rest of the diagram, indicated by the block $\beta$. 
summation over $L^{\prime \prime}$ and $S^{\prime \prime}$. It is to be emphasized that requiring zero angular momentum exchange between the $n p^{q-2}$ core and the rest of the diagram does not mean that there are no interactions between this core and $\phi_{a}$ or $\phi_{b}$ : one should remember that the block $\beta$ still includes such interactions. Since the rule in Figure 5 gives precisely the correct RPA interactions for a closed shell atom, we use Figure 5 to define our random phase approximation for an open-shell atom also.

\section{The coupled differential equations}

In Section 2 we have given our graphical procedures for evaluating the result of integrating equation (3) over $N-1$ coordinates using the initial and final states given in equations (4) and (5). We have still to obtain a set of equations which will determine the final radial functions $\psi^{\Gamma} \epsilon_{l^{\prime} \tilde{L}^{\prime}} \tilde{S}^{\prime}$ and a linear combination of $\phi_{a}$ and $\phi_{b}$ needed to obtain the matrix elements in equation (2). To obtain these equations, we note that each of the diagrams in Figures 1(a), 2(a), and 2(b) involves, by the rule in Figure 4 , a linear combination of double tensor operators $\left(l^{\prime}, \hat{r}^{\prime}\left|W^{\lambda, \sigma}\right| p, \hat{r}\right)$. The diagrams in Figures 1(b), 3(a), 3(b), and $3(\mathrm{c})$, on the other hand, involve a linear combination of double tensor operators $\left(p, \hat{r}^{\prime}\left|W^{\lambda, \sigma}\right| l^{\prime}, \hat{r}\right)$. For each $\lambda$ and $\sigma$, these two double tensors are linearly independent. Finally, by using the orthogonality properties of the 3j-symbols in Figure 4 , we may, for each $\lambda$ and $\sigma$, set the coefficients of the two double tensors above equal to zero.

The resulting pair of equations, for each set of values of $\lambda$, $\sigma$, and $l^{\prime}$, are sufficient to determine the radial wave functions we need in the closed shell case [11,13]. For open-shell atoms, however, these equations still have summations over certain quantum numbers (for example, the quantum numbers $\tilde{L}$ and $S$ of the $n p^{q-1}$ core [see Figures 1(a), 2(a), and 3(a)]), and thus we have fewer equations than we have radial functions to determine. This problem is overcome, however, by multiplying by an appropriate pair of $6 j$-coefficients and summing over $\lambda$ and $\sigma$ in order to project out exactly as many equations as there are radial functions to determine.

Resulting from these rather cumbersome but straightforward operations are the following coupled differential equations, in which for simplicity we have only indicated the interactions of the excited electrons with the open subshell:

$$
\begin{aligned}
& \left(-\frac{1}{2} \frac{\mathrm{d}^{2}}{\mathrm{~d} r^{2}}-\frac{Z}{r}+\frac{1}{2} \frac{l^{\prime}\left(l^{\prime}+1\right)}{r^{2}}-\epsilon_{n p}-\hbar \omega\right) \psi_{\epsilon l^{\prime} \widetilde{L} \widetilde{S}}^{\Gamma}(r) \\
& + \text { (interaction with closed subshells) } \\
& +\sum_{l^{\prime \prime}} \sum_{\widetilde{L}^{\prime} \widetilde{S}^{\prime}} \sum_{k}\left[A\left(l^{\prime}\left\|V^{k}\left(\chi_{n p}, \chi_{n p} ; r\right)\right\| l^{\prime \prime}\right) \psi_{\epsilon l^{\prime \prime} \widetilde{L}^{\prime} \widetilde{S}^{\prime}}^{\Gamma^{\prime}}(r)\right. \\
& \left.+B\left(l^{\prime}\left\|V^{k}\left(\chi_{n p}, \psi_{\epsilon l^{\prime \prime} \widetilde{L}^{\prime} \widetilde{s}^{\prime}} ; r\right)\right\| 1\right) \chi_{n p}(r)\right] \\
& +\sum_{l_{\phi} \Lambda \Sigma} \sum_{k} \sum_{k} C\left(l^{\prime}\left\|V^{k}\left(\Phi_{l_{\phi} \Lambda \Sigma}^{\Gamma}, \chi_{n p} ; r\right)\right\| 1\right) \chi_{n p}(r)=0 \\
& \left(-\frac{1}{2} \frac{\mathrm{d}^{2}}{\mathrm{~d} r^{2}}-\frac{Z}{r}+\frac{1}{2} \frac{l_{\phi}\left(l_{\phi}+1\right)}{r^{2}}-\epsilon_{n p}+\hbar \omega\right) \Phi_{l_{\phi} \Lambda \Sigma}^{\Gamma}(r) \\
& + \text { (interaction with closed subshells) } \\
& +\sum_{l_{\phi}^{\prime}} \sum_{\Lambda^{\prime} \Sigma^{\prime}} \sum_{k}\left[D\left(l_{\phi}^{\prime}|| V^{k}\left(\chi_{n p}, \chi_{n p} ; r\right)|| l_{\phi}\right) \Phi_{l_{\phi}^{\prime} \Lambda^{\prime} \Sigma^{\prime}}^{\Gamma}(r)\right. \\
& \left.+E\left(1\left\|V^{k}\left(\Phi_{l_{\phi}^{\prime} \Lambda^{\prime} \Sigma^{\prime}}^{\Gamma}, \chi_{n p} ; r\right)\right\| l_{\phi}\right) \chi_{n p}(r)\right] \\
& +\sum_{l^{\prime \prime} \widetilde{L}^{\prime} \widetilde{S}^{\prime}} \sum_{k} F\left(1\left\|V^{k}\left(\chi_{n p}, \psi_{\epsilon l^{\prime \prime} \widetilde{L}^{\prime} \widetilde{S}^{\prime}} ; r\right)\right\| l_{\phi}\right) \chi_{n p}(r)=0
\end{aligned}
$$

The coefficients $A, B, C, D, E$, and $F$ appearing in equations (9) and (10), as well as the linear combination of $\phi_{a}$ and $\phi_{b}$ denoted by $\Phi_{l_{\phi} \Sigma \Sigma}^{\Gamma}$, are defined in the Appendix. (The orbital and spin quantum numbers $\Lambda$ and $\Sigma$ may assume all values consistent with the triangular relations for certain $6 j$-coefficients appearing in equation (A9).) Note that the coefficients $A$ and $B$ are precisely the direct and exchange coefficients, respectively, of the close-coupling approximation [12]. Thus, in the absence of ground state correlations, equation (9) reduces to the closecoupling equations. Furthermore, we note that in the closedshell case there is only a single value for the quantum numbers $\tilde{L} \tilde{S}$ and $\Lambda \Sigma$. In this case our equations (9) and (10) as well as our functions $\Phi_{l_{\phi \Lambda \Sigma}}^{\Gamma}$ reduce to those of the Chang-Fano version of the RPA $[11,13]$. The interactions with closed subshells, indicated in equations (9) and (10), will be given elsewhere [14]. Finally, once equations (9) and (10) are solved for the radial functions $\psi^{\Gamma} \epsilon_{l^{\prime} \tilde{L}} \tilde{S}(r)$ and $\Phi_{l_{\phi} \Lambda \Sigma}^{\Gamma}(r)$ one may calculate the matrix elements of any one-body operator. In particular, the reduced radial dipole matrix elements are given by

$$
D_{\epsilon}^{\Gamma}=\sum_{l \widetilde{L} \widetilde{S}} G\left\langle\chi_{n p}\left\|r^{(1)}\right\| \psi_{\epsilon l \widetilde{L} \widetilde{S}}^{\Gamma}\right\rangle+\sum_{l_{\phi} \Lambda \Sigma} H\left\langle\Phi_{l_{\phi} \Lambda \Sigma}^{\Gamma}\left\|r^{(1)}\right\| \chi_{n p}\right\rangle
$$

Again, we have given the coefficients $G$ and $H$ in the Appendix. The reduced radial matrix elements are defined by

$$
\left\langle\psi_{a}\left\|r^{(1)}\right\| \psi_{b}\right\rangle \equiv(-1)^{l_{a}-l}>l_{>}^{1 / 2} \int_{0}^{\infty} \psi_{a}(r) r \psi_{b}(r) \mathrm{d} r
$$

where $l_{>}=\max \left(l_{a^{\prime}} l_{b}\right)$.

\section{Acknowledgments}

We wish to acknowledge support of this research by the U.S. Department of Energy under Contract No. EY-76-S-02-2892. A. F. Starace also wishes to acknowledge support of the Alfred P. Sloan Foundation. S. Shahabi would like to thank Professor K.-N. Huang for discussions concerning the graphical treatment of covariant and contravariant state vectors. A. F. Starace would like to thank Professors T. N. Chang and U. Fano for discussions concerning their derivation of RPA equations for closed shell atoms.

\section{References}

1. Amusia, M. Ya, and Cherepkov, N. A., Case Studies in Atomic Physics 5, 47 (1975).

2. Wendin, G., Photoionization and Other Probes of Many-Electron Interactions (Edited by F. J. Wuilleumier), p. 61, Plenum Press, New York, 1976.

3. Kelly, H. P., Photoionization and Other Probes of Many-Electron Interactions (Edited by F. J. Wuilleumier), p. 83, Plenum Press, New York, 1976.

4. Burke, P. G. and Robb, W. D., Adv. Atomic Mol. Phys. 11, 143 (1975).

5. Burke, P. G., and Taylor, K. T., J. Phys. B8, 2620 (1975).

6. Armstrong, L., J . Phys. B7,2320 (1974).

7. Dalgaard, E., J. Phys. B8, 695 (1975).

8. Starace, A. F., and Armstrong, L., Phys. Rev. A13, 1850 (1976).

9. Cherepkov, N. A., and Chernysheva, L. V., Phys. Letters 60A, 103 (1977); Izvestia Akadernii Nauk USSR 41, 2518 (1977).

10. Lowdin, P. O., Phys. Rev. 97, 1474 (1955).

11. Chang, T. N., and Fano, U., Phys. Rev. A13, 263 (1976); Phys. Rev. A13, 282 (1976).

12. Smith, K., Henry, R. J. W., and Burke, P. G., Phys. Rev. 147, 21 (1966).

13. Chang, T. N., Phys. Rev. A18, 1448 (1978).

14. Starace, A. F., and Shahabi, S., Phys. Rev. A (to be submitted).

15. Briggs, J. S., Rev. Mod. Phys. 43, 189 (1971).

16. Yutsis, A. P., Levinson, I. B., and Vanagas, V. V., The Theory of Angular Momentum, Israel Program for Scientific Translation, Jerusalem. 1962. 


\section{Appendix}

The coefficients introduced in equation (9) are:

$$
\begin{aligned}
& A \equiv \sum_{\bar{L} \bar{S}}(-1)^{1+\bar{L}+\widetilde{L}+\widetilde{L}^{\prime}+L_{f}}\left(p^{q-1} \widetilde{L} \widetilde{S}\left\{\mid p^{q-2} \bar{L} \bar{S}\right)\left(p^{q-2} \bar{L} \bar{S} \mid\right\} p^{q-1} \widetilde{L} \widetilde{S}^{\prime}\right) \\
& \times(q-1)[\tilde{L}]^{1 / 2}\left[\tilde{L}^{\prime}\right]^{1 / 2} \delta \widetilde{S} \widetilde{S}^{\prime}\left\{\begin{array}{lll}
1 & \tilde{L} & \bar{L} \\
\tilde{L}^{\prime} & 1 & k
\end{array}\right\}\left\{\begin{array}{lll}
L_{f} & l^{\prime \prime} & \tilde{L}^{\prime} \\
k & \tilde{L} & l^{\prime}
\end{array}\right\} \\
& B \equiv \sum_{\bar{L} \bar{S}}(-1)^{1-\widetilde{S}-\widetilde{S}^{\prime}}\left(p^{q-1} \tilde{L} \widetilde{S}\left\{\mid p^{q-2} \bar{L} \bar{S}\right)\left(p^{q-2} \bar{L} \bar{S} \mid\right\} p^{q-1} \widetilde{L}^{\prime} \widetilde{S}^{\prime}\right) \\
& \times(q-1)[\tilde{L}]^{1 / 2}\left[\tilde{L}^{\prime}\right]^{1 / 2}[\tilde{S}]^{1 / 2}\left[\tilde{S}^{\prime}\right]^{1 / 2} \\
& \times\left\{\begin{array}{ccc}
\bar{L} & 1 & \tilde{L} \\
1 & k & l^{\prime} \\
\tilde{L}^{\prime} & l^{\prime \prime} & L_{f}
\end{array}\right\}\left\{\begin{array}{ccc}
\tilde{S} & S_{f} & \frac{1}{2} \\
\widetilde{S}^{\prime} & \bar{S} & \frac{1}{2}
\end{array}\right\} \\
& C \equiv \frac{[\tilde{L}][\tilde{S}][\Lambda][\Sigma](-1)^{\tilde{L}+l_{\phi}+1 / 2+\widetilde{S}+k+q / 2}}{q^{1 / 2}\left(p^{q} L_{i} S_{i}\left\{\mid p^{q-1} \widetilde{L} \tilde{S}\right)\left[\frac{1}{2}\right]^{1 / 2}\left[l_{\phi}\right]^{1 / 2}\right.}
\end{aligned}
$$

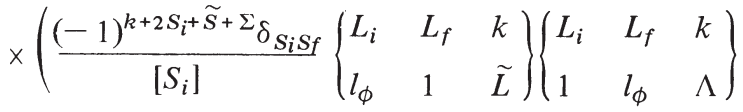

$$
\begin{aligned}
& +\frac{\delta \widetilde{S} \Sigma}{[\widetilde{S}]} \sum_{\lambda}[\lambda](-1)^{\lambda}\left\{\begin{array}{ccc}
L_{f} & \lambda & L_{i} \\
1 & \widetilde{L} & l_{\phi}
\end{array}\right\}\left\{\begin{array}{ccc}
l_{\phi} & \lambda & 1 \\
l^{\prime} & k & 1
\end{array}\right\} \\
& \left.\times\left\{\begin{array}{ccc}
1 & \lambda & l_{\phi} \\
L_{i} & \Lambda & L_{f}
\end{array}\right\}\right)
\end{aligned}
$$

The coefficients introduced in equation (10) are:

$$
\begin{aligned}
& D \equiv \delta_{\Sigma \Sigma^{\prime}}\left[\Lambda^{\prime}\right] \\
& \times\left(2\left(\frac{3}{5}\right)^{1 / 2} \sum_{\lambda}[\lambda]\left\{\begin{array}{ccc}
l_{\phi} & 1 & \lambda \\
L_{f} & L_{i} & \Lambda
\end{array}\right\}\left\{\begin{array}{ccc}
l_{\phi}^{\prime} & 1 & \lambda \\
L_{f} & L_{i} & \Lambda^{\prime}
\end{array}\right\} \delta_{k 0}\right. \\
& +(-1)^{l_{\phi^{+}} l_{\phi^{+}}^{\prime} L_{i}+L_{f}+\Lambda+\Lambda^{\prime}} \\
& \left.\times\left\{\begin{array}{ccc}
\Lambda^{\prime} & k & \Lambda \\
l_{\phi} & L_{i} & l_{\phi}^{\prime}
\end{array}\right\}\left\{\begin{array}{ccc}
\Lambda^{\prime} & k & \Lambda \\
l_{0} & L_{f} & 1
\end{array}\right\}\right) \\
& E \equiv\left[\Lambda^{\prime}\right]\left[\Sigma^{\prime}\right] \\
& \times\left((-1)^{1+\Sigma-\Sigma^{\prime}} \delta_{S_{i} S_{f}}\left[S_{i}\right]^{-1}\left\{\begin{array}{ccc}
l_{\phi} & 1 & k \\
L_{f} & L_{i} & \Lambda
\end{array}\right\}\left\{\begin{array}{ccc}
l_{\phi}^{\prime} & 1 & k \\
L_{f} & L_{i} & \Lambda^{\prime}
\end{array}\right\}\right. \\
& \left.+\frac{(-1)^{k+1} \delta_{\Sigma \Sigma^{\prime}}}{5[\Sigma]} \sum_{\lambda}[\lambda]\left\{\begin{array}{ccc}
l_{\phi} & 1 & \lambda \\
L_{f} & L_{i} & \Lambda
\end{array}\right\}\left\{\begin{array}{ccc}
l_{\phi}^{\prime} & 1 & \lambda \\
L_{f} & L_{i} & \Lambda^{\prime}
\end{array}\right\}\right)
\end{aligned}
$$

$$
\begin{aligned}
& F \equiv 2^{-1 / 2}[\widetilde{L}]^{1 / 2}\left[\widetilde{L}^{\prime}\right]^{1 / 2}[\check{S}]^{1 / 2}\left[\widetilde{S}^{\prime}\right]^{1 / 2}\left[l_{\phi}\right]^{1 / 2} q^{1 / 2}(q-1) \\
& \times \sum_{\tilde{L} \widetilde{S}}\left(n p ^ { q } L _ { i } S _ { i } \{ | n p ^ { q - 1 } \widetilde { L } \widetilde { S } ) \left(n p^{q-1} \widetilde{L} \widetilde{S}\left\{\mid n p^{q-2} \bar{L} \bar{S}\right)\right.\right. \\
& \left.\times\left(n p^{q-2} \bar{L} \bar{S} \mid\right\} n p^{q-1} \tilde{L}^{\prime} \tilde{S}^{\prime}\right)[\tilde{L}]^{1 / 2}[\tilde{S}]^{1 / 2} \\
& \times\left[(-1)^{l} \phi^{+} \tilde{L}+k+\Sigma-1 / 2-S_{i}-\bar{S}-\widetilde{S}-\widetilde{S}^{\prime}-q / 2\right. \\
& \times\left\{\begin{array}{ccc}
\frac{1}{2} & \bar{S} & \Sigma \\
\frac{1}{2} & S_{i} & \widetilde{S}
\end{array}\right\}\left\{\begin{array}{ccc}
\frac{1}{2} & \bar{S} & \Sigma \\
\frac{1}{2} & S_{f} & \widetilde{S}^{\prime}
\end{array}\right\}\left\{\begin{array}{ccc}
\bar{L} & l^{\prime \prime} & \Lambda \\
L_{f} & 1 & \tilde{L}^{\prime}
\end{array}\right\}\left\{\begin{array}{ccc}
L_{i} & 1 & \tilde{L} \\
l_{\phi} & k & 1 \\
\Lambda & l^{\prime \prime} & \bar{L}
\end{array}\right\} \\
& +(-1)^{l} \phi^{+} L_{i}+\widetilde{L}+\widetilde{L}^{\prime}+\bar{L}+\Lambda-2 \Sigma+1 / 2-\widetilde{S}^{\prime}-q / 2 \\
& \times\left\{\begin{array}{ccc}
L_{i} & l_{\phi} & \Lambda \\
k & \widetilde{L} & 1
\end{array}\right\}\left\{\begin{array}{ccc}
\widetilde{L} & k & \Lambda \\
l^{\prime \prime} & \bar{L} & 1
\end{array}\right\}\left\{\begin{array}{ccc}
\bar{L} & l^{\prime \prime} & \Lambda \\
L_{f} & 1 & \widetilde{L}^{\prime}
\end{array}\right\} \\
& \left.\times \frac{\delta_{\Sigma \widetilde{S}}}{[\Sigma]}\left\{\begin{array}{ccc}
\frac{1}{2} & \bar{S} & \Sigma \\
\frac{1}{2} & S_{f} & \widetilde{S}^{\prime}
\end{array}\right\}\right]
\end{aligned}
$$

The coefficients $G$ and $H$ introduced in equation (11) are given by:

$$
\begin{aligned}
& G \equiv(-1)^{L+L_{i}+1} q^{1 / 2}\left(p^{q} L_{i} S_{i}\left\{\mid p^{q-1} \tilde{L} \tilde{S}\right)\left[L_{i}\right]^{1 / 2}\left[L_{f}\right]^{1 / 2} \delta_{S_{i} S_{f}}\right. \\
& \times\left\{\begin{array}{lll}
L_{i} & L_{f} & 1 \\
l & 1 & \tilde{L}
\end{array}\right\} \\
& H \equiv(-1)^{L_{i}+l_{\phi^{+1 / 2}-\Sigma+q / 2}} \frac{\left[L_{f}\right]^{1 / 2}\left[L_{i}\right]^{1 / 2}[\Lambda][\Sigma]}{\left[l_{\phi}\right]^{1 / 2}\left[\frac{1}{2}\right]^{1 / 2}}\left\{\begin{array}{lll}
L_{i} & L_{f} & 1 \\
1 & l_{\phi} & \Lambda
\end{array}\right\}
\end{aligned}
$$

Finally, the function $\Phi_{l_{\phi} \Lambda \Sigma}^{\Gamma}(r)$ introduced in equations (9)-(11) is defined in terms of the functions $\phi_{a}$ and $\phi_{b}$ of equation (5) as follows:

$$
\begin{aligned}
\Phi_{l_{\phi} \Lambda \Sigma}^{\Gamma} & \left.(r)=\sum_{\widetilde{L} \widetilde{S}} \sum_{\bar{L} \bar{S}} \sum_{L_{P} S_{P}} b_{\bar{L} \bar{S}, L_{P} S_{P}}\left(p^{q-2} \overline{L S} \mid\right\} p^{q-1} \tilde{L} \widetilde{S}\right) \\
& \times(q-1)^{1 / 2}\left[\frac{1}{2}\right]^{1 / 2}\left[l_{\phi}\right]^{1 / 2}[\tilde{L}]^{1 / 2}\left[L_{P}\right]^{1 / 2}[\widetilde{S}]^{1 / 2}\left[S_{P}\right]^{1 / 2} \\
& \times(-1)^{L_{i}+L_{P}+\bar{L}+\widetilde{L}+1}\left\{\begin{array}{ccc}
l_{\phi} & \widetilde{L} & L_{f} \\
1 & \Lambda & \bar{L}
\end{array}\right\}\left\{\begin{array}{ccc}
\bar{L} & L_{P} & L_{i} \\
l_{\phi} & \Lambda & l_{\phi}
\end{array}\right\} \\
& \times(-1)^{-q / 2+\widetilde{S}+2 S_{f}+3 / 2+\bar{S}+S p-S_{i}}\left\{\begin{array}{ccc}
\frac{1}{2} & \widetilde{S} & S_{f} \\
\frac{1}{2} & \Sigma & \bar{S}
\end{array}\right\}\left\{\begin{array}{ccc}
\bar{S} & S_{P} & S_{i} \\
\frac{1}{2} & \Sigma & \frac{1}{2}
\end{array}\right\} \\
& \times\left[\left(\phi_{b} \mid \psi_{\epsilon l_{\phi} \tilde{L} \widetilde{S}}^{\Gamma}\right) \phi_{a}(r)+(-1)^{\left.L_{P}+S_{P}\left(\phi_{a} \mid \psi_{\epsilon l_{\phi}}^{\Gamma} \tilde{L}\right) \phi_{b}(r)\right]}\right. \text { (A9) }
\end{aligned}
$$

Note that we never need to determine the functions $\phi_{a}(r)$ and $\phi_{b}(r)$, but only the linear combination given by equation (A9). 